Claremont Colleges

Scholarship@ Claremont

All HMC Faculty Publications and Research

HMC Faculty Scholarship

$1-1-1982$

\title{
Rearrangeable Networks with Limited Depth
}

Nicholas Pippenger

Harvey Mudd College

Andrew C.-C. Yao

University of California - Berkeley

\section{Recommended Citation}

Pippenger, Nicholas, and Andrew C.-C. Yao. "Rearrangeable Networks with Limited Depth." SIAM Journal on Algebraic and Discrete Methods 3, no. 4 (December 1982): 411-417.

This Article is brought to you for free and open access by the HMC Faculty Scholarship at Scholarship @ Claremont. It has been accepted for inclusion in All HMC Faculty Publications and Research by an authorized administrator of Scholarship @ Claremont. For more information, please contact scholarship@cuc.claremont.edu. 


\title{
REARRANGEABLE NETWORKS WITH LIMITED DEPTH*
}

\author{
NICHOLAS PIPPENGER † AND ANDREW C.-C. YAO
}

\begin{abstract}
Rearrangeable networks are switching systems capable of establishing simultaneous independent communication paths in accordance with any one-to-one correspondence between their $n$ inputs and $n$ outputs. Classical results show that $\Omega(n \log n)$ switches are necessary and that $O(n \log n)$ switches are sufficient for such networks. We are interested in the minimum possible number of switches in rearrangeable networks in which the depth (the length of the longest path from an input to an output) is at most $k$, where $k$ is fixed as $n$ increases. We show that $\Omega\left(n^{1+1 / k}\right)$ switches are necessary and that $O\left(n^{1+1 / k}(\log n)^{1 / k}\right)$ switches are sufficient for such networks.
\end{abstract}

1. Introduction. An $(m, n)$-network $G=(V, E, A, B)$ comprises an acyclic directed graph with vertices $V$ and edges $E$, a set of $m$ distinguished vertices $A$ called inputs and a set of $n$ other distinguished vertices $B$ called outputs.

A request is an ordered pair $(a, b)$ comprising an input $a$ and an output $b$. A route is a directed path from an input to an output. A route satisfies a request $(a, b)$ if it is from $a$ to $b$.

An $l$-assignment is a set of $l$ requests, no two of which have an input or output in common. An $l$-state is a set of $l$ routes, no two of which have a vertex in common. An $l$-state satisfies an $l$-assignment if it contains a route satisfying each request in the assignment.

An $n$-connector (also known as a rearrangeable $n$-network) is an (n, n)-network that has an $n$-state satisfying each of the $n ! n$-assignments. The size of a network is the number of edges in it. The depth of a network is the maximum number of edges in any route in it.

Let $f(n)$ denote the minimum possible size of an $n$-connector. An informationtheoretic argument (due to C. E. Shannon) shows that $f(n)=\Omega(n \log n)$ (see Pippenger [4]; $\Omega(\cdots)$ means "some function bounded below by a strictly positive constant times ..."). A classical construction (due to D. Slepian, A. M. Duguid and J. LeCorre) shows that $f(n)=O(n \log n)$ (see Pippenger [3]; $O(\cdots)$ means "some function bounded above in absolute value by a constant times ...").

Let $f_{k}(n)$ denote the minimum possible size of an $n$-connector having depth at most $k$. We shall be interested in the behavior of $f_{k}(n)$ as $n$ grows while $k$ remains fixed. The case $k=1$ is trivial: $f_{1}(n)=n^{2}$. For $k=2$, a probabilistic argument (used by de Bruijn, Erdös and Spencer [1] to solve a problem of van Lint [2]) shows that $f_{2}(n)=O\left(n^{3 / 2}(\log n)^{1 / 2}\right)$. For odd $k \geqq 3$, the classical construction referred to above shows that $f_{k}(n)=O\left(n^{1+2 /(k+1)}\right)$.

In $\S 2$ we shall show (by adapting an argument due to Pippenger and Valiant [5]) that $f_{k}(n)=\Omega\left(n^{1+1 / k}\right)$. In $\S \S 3$ and 4 we shall show (by a probabilistic argument) that $f_{k}(n)=O\left(n^{1+1 / k}(\log n)^{1 / k}\right)$.

2. Lower bound. An $n$-tree is a $(1, n)$-network with inputs $A=\{a\}$, outputs $B=\left\{b_{1}, \cdots, b_{n}\right\}$ and, for $1 \leqq j \leqq n$, a unique route $R_{j}$ satisfying the request $\left(a, b_{j}\right)$.

If $T$ is an $n$-tree, let

$$
\Delta(T)=\sum_{1 \leqq j \leqq n} \sum_{v \in R_{i}} d_{v}
$$

* Received by the editors June 6, 1981.

† Computer Science Department, IBM Research Laboratory, San Jose, California 95193.

$\ddagger$ Department of Electrical Engineering and Computer Sciences, University of California, Berkeley, California 94720. 
where $d_{v}$ denotes the number of edges directed out of the vertex $v$.

Proposition 2.1. If $T$ is an $n$-tree of depth at most $k \geqq 1$, then

$$
\Delta(T) \geqq k n^{1+1 / k} \text {. }
$$

Proof. The proof is by induction on $k$. The case $k=1, \Delta(T)=n^{2}$ is trivial. If $k \geqq 2$, let $d$ be the number of edges directed out of the input and let $T_{1}, \cdots, T_{d}$ (with $n_{1}, \cdots, n_{d}$ outputs, respectively) be the subtrees into which these edges are directed.

We have

$$
\Delta(T)=d n+\sum_{1 \leqq h \leqq d} \Delta\left(T_{h}\right) \geqq d n+\sum_{1 \leqq h \leqq d}(k-1) n_{h}^{1+1 /(k-1)},
$$

by inductive hypothesis. Since $n_{1}+\cdots+n_{d}=n$ and $(k-1) \theta^{1+1 /(k-1)}$ is convex in $\theta$, we have

$$
\sum_{1 \leqq h \leqq d}(k-1) n_{h}^{1+1 /(k-1)} \geqq d(k-1)\left(\frac{n}{d}\right)^{1+1 /(k-1)} .
$$

Straightforward calculus shows that

$$
d n+d(k-1)\left(\frac{n}{d}\right)^{1+1 /(k-1)} \geqq k n^{1+1 / k},
$$

which completes the induction.

An $n$-shifter is an $(n, n)$-network with inputs $A=\left\{a_{1}, \cdots, a_{n}\right\}$, outputs $B=$ $\left\{b_{1}, \cdots, b_{n}\right\}$ and, for $1 \leqq j \leqq n$, a state satisfying the assignment $\left\{\left(a_{1}, b_{j+1}\right), \cdots,\left(a_{n}, b_{j+n}\right)\right\}$ (addition is modulo $n$ ).

THEOREM 2.1. Any $n$-shifter of depth at most $k$ has size at least $k n^{1+1 / k}$.

Proof. Let $G=(V, E, A, B)$ be an $n$-shifter of depth at most $k$. Let $R_{i, j}$ be the route from input $a_{i}$ to output $b_{j+i}$ in the state that satisfies the assignment $\left\{\left(a_{1}, b_{j+1}\right), \cdots,\left(a_{n}, b_{j+n}\right)\right\}$. By identifying common initial segments, the routes $R_{i, 1}, \cdots, R_{i, n}$ can be assembled into an $n$-tree $T_{i}$ of depth at most $k$, for which

$$
\Delta\left(T_{i}\right) \geqq k n^{1+1 / k},
$$

by Proposition 2.1. For $1 \leqq i \leqq n, 1 \leqq j \leqq n$ and $e \in E$, let $\mu(i, j, e)$ be 1 if the edge $e$ is directed out of a vertex on $R_{i, j}$ and 0 otherwise. For any $i$ and $j$, we have

$$
\sum_{e \in E} \mu(i, j, e) \geqq \sum_{v \in R_{i, j}} d_{v}
$$

and by summing over $j$, we have

$$
\sum_{1 \leqq j \leqq n} \sum_{e \in E} \mu(i, j, e) \geqq \sum_{1 \leqq j \leqq n} \sum_{v \in R_{i, j}} d_{v} \geqq \Delta\left(T_{i}\right) \geqq k n^{1+1 / k} .
$$

Thus,

$$
\sum_{1 \leqq i \leqq n} \sum_{1 \leqq j \leqq n} \sum_{e \in E} \mu(i, j, e) \geqq k n^{2+1 / k} .
$$

On the other hand, since the routes $R_{1, j}, \cdots, R_{n, j}$ have no vertex in common, an edge $e$ can be directed out of a vertex on at most one of them. Thus, for any $j$ and $e$, we have

$$
\sum_{1 \leqq i \leqq n} \mu(i, j, e) \leqq 1
$$


By summing over $j$ we have

$$
\sum_{1 \leqq j \leqq n} \sum_{1 \leqq i \leqq n} \mu(i, j, e) \leqq n,
$$

and by summing over $e$ we have

$$
\sum_{e \in E} \sum_{1 \leqq j \leqq n} \sum_{1 \leqq i \leqq n} \mu(i, j, e) \leqq n \#(E)
$$

(\# $(\cdots)$ means "the cardinality of $\cdots$ "). Comparing this with $(2.1)$ gives

$$
\#(E) \geqq k n^{1+1 / k} \text {, }
$$

as claimed.

Corollary 2.1. Any $n$-connector of depth at most $k$ has size at least $k n^{1+1 / k}$.

Proof. An $n$-connector is an $n$-shifter.

3. Couplers. A set $\mathscr{X}=\left\{X_{1}, \cdots, X_{r}\right\}$ is an $x$-packing of a set $A$ if $X_{1}, \cdots, X_{r}$ are mutually disjoint $x$-element subsets of $A$. An $x$-packing $\mathscr{X}$ of $A$ is tight if $\#(\bigcup \mathscr{X}) \geqq$ $\#(A) / 16$ (or, equivalently, \# $(\mathscr{X}) \geqq \#(A) / 16 x$ ).

If $G$ is a network and $X$ a set of inputs of $G$, let $G(X)$ denote the set of outputs of $G$ reachable through routes from inputs in $X$.

An $(l, l)$-networks $G=(V, E, A, B)$ is an $(l, x, y)$-coupler if, for every tight $x$ packing $\mathscr{X}=\left\{X_{1}, \cdots, X_{r}\right\}$ of $A$, there exists a tight $y$-packing $\mathscr{Y}=\left\{Y_{1}, \cdots, Y_{s}\right\}$ of $B$ such that, for every $1 \leqq j \leqq s$, there exists $1 \leqq i \leqq r$ such that $Y_{j} \subseteq G\left(X_{i}\right)$.

If $G$ is an $(l, m)$-network and $H$ is an $(m, n)$-network, let $G \circ H$ denote an $(l, n)$-network obtained by identifying the outputs of $G$ with the inputs of $H$ in any one-to-one fashion (to become vertices that are neither inputs nor outputs of $G \circ H$ ).

LEMMA 3.1. If $G$ is an $(l, x, y)$-coupler and $H$ is an $(l, y, z)$-coupler, then $G \circ H$ is an $(l, x, z)$-coupler.

Proof. The proof is immediate.

An $(m, m)$-network $G$ is a strong $(m, x, y)$-coupler if, for every $m / 2 \leqq l \leqq m$, each $(l, l)$-network obtained from $G$ by deleting $m-l$ vertex-disjoint routes (together with all edges incident with vertices on these routes) is an $(l, x, y)$-coupler.

LEMMA 3.2. If $G$ is a strong ( $m, x, y)$-coupler and $H$ is a strong ( $m, y, z)$-coupler, then $G \circ H$ is a strong $(m, x, z)$-coupler.

Proof. The proof follows from Lemma 3.1.

LEMMA 3.3. Let $\mathbf{X}$ denote the number of successes among $n$ trials that succeed independently with probability $p$. Then

$$
\mathscr{P}(\mathbf{X}>2 n p) \leqq\left(\frac{e}{4}\right)^{n p}
$$

and

$$
\mathscr{P}\left(\mathbf{X}<\frac{n p}{2}\right) \leqq\left(\frac{2}{e}\right)^{n p / 2}
$$

$(\mathscr{P}(\cdots)$ means "the probability of $\cdots$. .").

Proof. For (3.1), we may assume $p<\frac{1}{2}$, for otherwise $\mathscr{P}(\mathbf{X}>2 n p)=0$. If

$$
\mathbf{Y}= \begin{cases}0 & \text { if } \mathbf{X} \leqq 2 n p \\ 1 & \text { if } \mathbf{X}>2 n p\end{cases}
$$

then $\mathscr{P}(\mathbf{X}>2 n p)=\mathscr{E}(\mathbf{Y})$. If $\mathbf{Z}=T^{\mathbf{X}-2 n p}$ (where $T>1$ is a parameter to be chosen later), then $\mathbf{Y} \leqq \mathbf{Z}$ and so $\mathscr{E}(\mathbf{Y}) \leqq \mathscr{E}(\mathbf{Z})$. Thus it will suffice to estimate $\mathscr{E}(\mathbf{Z})$. 
Since $\mathbf{X}$ is the sum of $n$ independent random variables that assume the value 1 with probability $p$ and the value 0 with probability $1-p, T^{\mathbf{X}}$ is the product of $n$ independent random variables that have expected value $p T+1-p$. Thus,

$$
\mathscr{E}(\mathbf{Z})=(p T+1-p)^{n} T^{-2 n p} .
$$

Choosing $T=2(1-p) /(1-2 p)$ and using the inequality $1+\theta \leqq e^{\theta}$ yields (3.1). A similar argument yields (3.2).

Proposition 3.1. If

$$
512 x \ln m \leqq y \leqq \frac{m}{16},
$$

then there exists a strong ( $m, x, y)$-coupler of depth 1 and size at most $32 m y / x$.

Proof. Let

$$
p=\frac{16 y}{x},
$$

and let $\mathbf{G}=(V, \mathbf{E}, A, B)$ be the random $(m, m)$-network $\mathbf{K}_{m, m}(p)$ (an $(m, m)$-network of depth 1 in which each of the $m^{2}$ potential edges is independently present with probability $p$ ). We expect $m^{2} p=16 m y / x$ edges in $\mathbf{E}$, so

$$
\mathscr{P}\left(\#(\mathbf{E})>\frac{32 m y}{x}\right) \leqq\left(\frac{e}{4}\right)^{16 m y / x} \leqq \frac{1}{4},
$$

by Lemma 3.3. Thus, it will suffice to show that

$$
\mathscr{P}(\mathbf{G} \text { not a strong }(m, x, y) \text {-coupler }) \leqq \frac{1}{4} .
$$

There are at most $4^{m}(l, l)$-networks $\mathbf{F}$ obtained from $\mathbf{G}$ by deleting $m-l$ vertexdisjoint routes (together with all edges incident with vertices on these routes). It will thus suffice to show for $m / 2 \leqq l \leqq m$ and $\mathbf{F}=\mathbf{K}_{l, l}(p)$ that

$$
\mathscr{P}(\mathbf{F} \text { not an }(l, x, y) \text {-coupler }) \leqq \frac{1}{4^{m+1}} .
$$

There are at most $l^{l} \leqq m^{m}$ minimal tight $x$-packings $\mathscr{X}=\left\{X_{1}, \cdots, X_{r}\right\}$ (where $r=\lceil l / 16 x\rceil)$ of the inputs of $\mathbf{F}$. Thus, it will suffice to show that

$$
\mathscr{P}(\mathbf{F} \text { not an }(l, x, y) \text {-coupler for } \mathscr{X}) \leqq \frac{1}{4^{m+1} m^{m}} .
$$

We shall consider each set $X_{i}$ in turn. For each set $X_{i}$, we shall attempt to construct a set $Y_{j}$ containing $y$ outputs, disjoint from all previously constructed sets $Y_{1}, \cdots, Y_{j-1}$ and satisfying $Y_{j} \subseteq \mathbf{F}\left(X_{i}\right)$. If we show that

$$
\mathscr{P}\left(\text { no } Y_{j} \text { for } X_{i}\right) \leqq\left(\frac{2}{e}\right)^{y},
$$

then the probability of fewer than $s=\lceil l / 16 y\rceil$ successes among $r$ trials will be at most

$$
\begin{aligned}
2^{r}\left[\left(\frac{2}{e}\right)^{y}\right]^{r-s} & \leqq 2^{r}\left[\left(\frac{2}{e}\right)^{y}\right]^{r / 2} \leqq\left[4\left(\frac{2}{e}\right)^{y}\right]^{l / 32 x} \leqq\left[4\left(\frac{2}{e}\right)^{y}\right]^{m / 64 x} \\
& \leqq 4^{m}\left(\frac{2}{e}\right)^{m y / 64 x} \leqq 4^{m} e^{-m y / 256 x} \leqq 4^{m} e^{-2 m \ln m} \leqq \frac{1}{4^{m+1} m^{m}}
\end{aligned}
$$


To construct $Y_{j}$, we shall consider each output of $\mathbf{F}$ that is not in $Y_{1} \cup \cdots \cup Y_{j-1}$ in turn (there are at least $l-s y \geqq l / 2 \geqq m / 4$ such outputs). For each such output, we shall attempt to find an edge joining it to an input in $X_{i}$. The probability of finding such an edge is

$$
1-(1-p)^{x} \geqq 1-e^{-p x} \geqq \frac{p x}{2}
$$

(using $1-\theta \leqq e^{-\theta} \leqq 1-\theta / 2$ for $\left.0 \leqq \theta \leqq 1\right)$. Thus, we expect at least $(m / 4)(p x / 2)=2 y$ successes and the probability of fewer than $y$ successes is at most $(2 / e)^{y}$, by Lemma 3.3.

COROllary 3.1. If

$$
512 \ln m \leqq x
$$

and

$$
x^{k-1} \leqq \frac{m}{16}
$$

then there exists a strong $\left(m, x, x^{k-1}\right)$-coupler of depth $k-2$ and size at most $32(k-2) m x$.

Proof. The proof follows from Lemma 3.2 and Proposition 3.1.

4. Upper bound. An $(n, n)$-network is an $(a, b)$-partial $n$-connector if, for every $a$-assignment $P$, there exists an $(a-b)$-assignment $Q \subseteq P$ and a state satisfying $Q$.

If $Q$ and $H$ are $(n, n)$-networks, let $G \| H$ denote an $(n, n)$-network obtained by identifying the inputs of $G$ with the inputs of $H$ in any one-to-one fashion (to become the inputs of $G \| H$ ) and identifying the outputs of $G$ with the outputs of $H$ in any one-to-one fashion (to become the outputs of $G \| H$ ).

LEMMA 4.1. If $G$ is an (a,b)-partial n-connector and $H$ is $a(b, c)$-partial $n$ connector, then $G \| H$ is an $(a, c)$-partial n-connector.

Proof. The proof is immediate.

LEMMA 4.2. Let $L$ be an l-element set and let $\mathscr{C}$ be a collection of subsets of $L$ such that if $Y \in \mathscr{C}$ and $X \subseteq Y$, then $X \in \mathscr{C}$. If $\mathscr{C}$ contains more than

$$
2^{-2 x}\left(\begin{array}{c}
l \\
2 x
\end{array}\right)
$$

(2x)-element subsets of $L$, then it contains a tight $x$-packing of $L$.

Proof. Let $\mathbf{Y}$ be a random uniformly distributed $(2 x)$-element subset of $L$, then

$$
\mathscr{P}(\mathbf{Y} \in \mathscr{C})>2^{-2 x} \text {. }
$$

Let $\mathscr{X}$ be a maximal $x$-packing contained in $\mathscr{C}$. If $\mathscr{X}$ is not tight, then

$$
\#(\bigcup \mathscr{X}) \leqq \frac{l}{16}
$$

and

$$
\mathscr{P}(\#(\mathbf{Y} \cap \bigcup \mathscr{X})>x) \leqq 2^{2 x} 16^{-x} \leqq 2^{-2 x} .
$$

Thus, there exists a $(2 x)$-element set $Y \in \mathscr{C}$ for which $\#(Y \cap \bigcup \mathscr{X}) \leqq x$. Then $Y-\bigcup \mathscr{X}$ contains an $x$-element set that can be added to $\mathscr{X}$ to yield a larger $x$-packing, contradicting the maximality of $\mathscr{X}$.

Proposition 4.1. If

$$
512^{k}(2 \ln 2 n)^{k-1} \leqq m \leqq n,
$$


then there exists an $(m, m / 2)$-partial $n$-connector of depth $k$ and size at most $64(k-1) \cdot$ $n(2 m \ln 2 n)^{1 / k}$.

Proof. Set

$$
x=\left\lceil(2 m \ln 2 n)^{1 / k}\right\rceil .
$$

Then $512 \ln m \leqq x$ and $x^{k-1} \leqq m / 16$, and by Corollary 3.1 , there exists a strong $\left(m, x, x^{k-1}\right)$-coupler $G$ of depth $k-2$ and size $32(k-2) m x$.

Let

$$
q=\frac{8 x}{m},
$$

and let $\mathbf{H}=(V, \mathbf{E}, A, B)$ be the random $(n, n)$-network $\mathbf{K}_{n, m}(q) \circ G \circ \mathbf{K}_{m, n}(q)$. We expect $2 n m q=16 n x$ edges in $\mathbf{K}_{n, m}(q)$ and $\mathbf{K}_{m, n}(q)$ together, so

$$
\mathscr{P}(\#(\mathbf{E}) \geqq 32(k-1) n x) \leqq\left(\frac{e}{4}\right)^{16 n x} \leqq \frac{1}{4},
$$

by Lemma 3.3. It will thus suffice to show that

$$
\mathscr{P}(\mathbf{H} \text { not an }(m, m / 2) \text {-partial } n \text {-connector }) \leqq \frac{1}{4} \text {. }
$$

There are at most $n^{2 m} / 4 m$-assignments $P$. Thus, it will suffice to show that

$$
\mathscr{P}(\mathbf{H} \text { not an }(m, m / 2) \text {-partial } n \text {-connector for } P) \leqq n^{-2 m} \text {. }
$$

We shall consider each of the $m$ requests in $P$ in turn. For each request $(a, b)$, we shall attempt to construct a route, vertex-disjoint from all previously constructed routes and satisfying the request $(a, b)$. If we show that

$$
\mathscr{P}(\text { no route for }(a, b)) \leqq \frac{1}{4 n^{4}},
$$

then the probability of fewer than $m / 2$ successes among $m$ trials will be at most

$$
2^{m}\left(\frac{1}{4 n^{4}}\right)^{m / 2}=n^{-2 m}
$$

The probability that there is no route for $(a, b)$ is the probability that there is no route in the random network $\mathbf{I}=\mathbf{K}_{1, l}(q) \circ F \circ \mathbf{K}_{l, 1}(q)$, where $m / 2 \leqq l \leqq m$ and $F$ is an $\left(l, x, x^{k-1}\right)$-coupler. Let $\xi$ denote the random number of outputs of $\mathbf{K}_{1, l}(q)$ reachable through routes from the input of $\mathbf{K}_{1, l}(q)$, let $\eta$ denote the random number of outputs of $\mathbf{K}_{1, l}(q) \circ F$ reachable through routes from the input of $\mathbf{K}_{1, l}(q) \circ F$ and let $\zeta$ denote the random number of inputs of $\mathbf{K}_{l, 1}(q)$ from which the output of $\mathbf{K}_{l, 1}(q)$ is reachable. Then

$$
\begin{aligned}
\mathscr{P}(\text { no route }) \leqq & \mathscr{P}\left(\text { no route } \mid \eta \geqq x^{k-1}, \zeta \geqq 2 x\right)+\mathscr{P}\left(\eta<x^{k-1}\right)+\mathscr{P}(\zeta<2 x) \\
\leqq & \mathscr{P}\left(\text { no route } \mid \eta \geqq x^{k-1}, \zeta \geqq 2 x\right)+\mathscr{P}\left(\eta<x^{k-1} \mid \xi \geqq 2 x\right)+\mathscr{P}(\xi<2 x) \\
& +\mathscr{P}(\zeta<2 x) .
\end{aligned}
$$

The random variables $\xi$ and $\zeta$ have expected value $l q \geqq m q / 2=4 x$, so

$$
\mathscr{P}(\xi<2 x)=\mathscr{P}(\zeta<2 x) \leqq\left(\frac{2}{e}\right)^{2 x} \leqq \frac{1}{16 n^{4}},
$$


by Lemma 3.3. Furthermore,

$$
\begin{aligned}
\mathscr{P}\left(\text { no route } \mid \eta \geqq x^{k-1}, \zeta \geqq 2 x\right) & \leqq\left(\begin{array}{c}
l-x^{k-1} \\
2 x
\end{array}\right) /\left(\begin{array}{c}
l \\
2 x
\end{array}\right) \\
& \leqq e^{-2 x^{k / l}} \leqq e^{-2 x^{k / m}} \leqq e^{-4 \ln 2 n} \leqq \frac{1}{16 n^{4}}
\end{aligned}
$$

Thus, it will suffice to show that $\mathscr{P}\left(\eta<x^{k-1} \mid \xi \geqq 2 x\right) \leqq 1 / 16 n^{4}$.

Let $\mathscr{C}$ be the collection of subsets $X$ of the inputs of $F$ for which $\#(F(X))<x^{k-1}$. Since $F$ is an $\left(l, x, x^{k-1}\right)$-coupler, $\mathscr{C}$ contains no tight $x$-packing and thus, by Lemma $4.2, \mathscr{C}$ contains at most

$$
2^{-2 x}\left(\begin{array}{c}
l \\
2 x
\end{array}\right)
$$

(2x)-element subsets. Thus,

$$
\mathscr{P}\left(\eta<x^{k-1} \mid \xi \geqq 2 x\right) \leqq 2^{-2 x} \leqq \frac{1}{16 n^{4}},
$$

as was to be shown.

COROLlARY 4.1. If

$$
b=512^{k}(2 \ln 2 n)^{k-1},
$$

then there exists an $(n, b)$-partial $n$-connector of depth $k$ and size at most

$$
256 k(k-1) n(2 n \ln 2 n)^{1 / k} \text {. }
$$

Proof. The result follows from Lemma 4.1, Proposition 4.1 and

$$
\sum_{1 \leqq j<\infty} 2^{-j / k}=\frac{1}{\left(1-2^{1 / k}\right)} \leqq 4 k
$$

(using $e^{-\theta} \leqq 1-\theta / 2$ for $0 \leqq \theta \leqq 1$ ).

LemMA 4.3. There exists an (a,0)-partial n-connector of depth 2 and size $2 a n$.

Proof. Consider $K_{n, a} \circ K_{a, n}$.

THEOREM 4.1. There exists an $n$-connector of depth $k$ and size at most

$$
256 k(k-1) n(2 n \ln 2 n)^{1 / k}+2(512)^{k} n(2 \ln n)^{k-1} \text {. }
$$

Proof. The result follows from Lemma 4.1, Corollary 4.1 and Lemma 4.3; an $(n, 0)$-partial $n$-connector is an $n$-connector.

\section{REFERENCES}

[1] N. G. DE Bruijn, P. Erdös AND J. Spencer, Solution 350, Nieuw Archief voor Wiskunde, 22 (1974), p. 94-109.

[2] J. H. VAN Lint, Problem 350, Nieuw Archief voor Wiskunde, 21 (1973), p. 179.

[3] N. PIPPENGER, On rearrangeable and non-blocking switching networks, J. Comput. System Sci., 17, (1978), pp. 145-162.

[4] — A new lower bound for the number of switches in rearrangeable networks, this Journal, 1 (1980), pp. 164-167.

[5] N. Pippenger And L. G. Valiant, Shifting graphs and their applications, J. Assoc. Comput. Mach., 23 (1976), pp. 423-432. 